\title{
Relação entre níveis de atividade física, variáveis relacionadas à multidimensionalidade da saúde e presença de estresse percebido em um grupo de
}

\section{idosos na cidade de São Paulo}

\author{
Relationship between levels of physical activity, variables related to the multidimensionality of \\ health and the presence of perceived stress in a group of elderly in the city of São Paulo \\ Relación entre niveles de actividad física, variables relacionadas con la multidimensionalidad de la \\ salud y presencia de estrés percibido en un grupo de personas mayores en la cuidad de São Paulo
}

Recebido: 30/12/2020 | Revisado: 31/12/2020 | Aceito: 02/01/2021 | Publicado: 05/01/2021

\author{
Maria Elisa Gonzalez Manso \\ ORCID: https://orcid.org/0000-0001-5446-233X \\ Centro Universitário São Camilo, Brasil \\ E-mail: mansomeg@hotmail.com \\ Renata Fonseca Inácio Osti \\ ORCID: https://orcid.org/0000-0002-7253-500X \\ Centro Universitário São Camilo, Brasil \\ E-mail: renata.osti@prof.saocamilo-sp.br \\ Diana Santana Moura Fé \\ ORCID: https://orcid.org/0000-0003-2730-914X \\ Centro Universitário São Camilo, Brasil \\ E-mail: dianasmourafe@gmail.com \\ Fabiana Corrêa Bonadia de Souza \\ ORCID: https://orcid.org/0000-0002-1979-9671 \\ Centro Universitário São Camilo, Brasil \\ E-mail: fabibonadiaa@gmail.com \\ Isabella Ferreira Pimenta \\ ORCID: https://orcid.org/0000-0002-5375-7694 \\ Centro Universitário São Camilo, Brasil \\ E-mail: isafpim@gmail.com \\ Luiza Travassos da Rosa Netto \\ ORCID: https://orcid.org/0000-0003-2707-1944 \\ Centro Universitário São Camilo, Brasil \\ E-mail: luizatrnetto@gmail.com
}

\begin{abstract}
Resumo
Trata-se de pesquisa realizada com um grupo de pessoas idosas que participam ativamente de um Núcleo de Convivência para Idosos situado na Zona Leste de São Paulo. O objetivo foi verificar a associação entre os níveis de prática de atividade física e variáveis relacionadas tanto à multidimensionalidade da saúde na pessoa idosa quanto ao grau de estresse percebido. Investigou-se, ainda, quais das variáveis estudadas influenciaram os níveis de atividade física encontrados neste grupo. Participaram 57 idosos, em sua maioria mulheres, viúvas ou casadas, com baixa escolaridade e média de idade de 74,7 anos, independentes para o desempenho de atividades básicas e instrumentais de vida diária, sem risco de queda ou cognitivo. Estas pessoas idosas, de maneira geral, autoperceberam sua saúde como boa ou ótima, sendo fisicamente ativas. Encontrou-se associação estatisticamente significante entre a presença de sintomas depressivos e elevados níveis de estresse percebido, notando-se que idosos irregularmente ativos apresentaram mais sintomas depressivos e pior autopercepção de saúde. Para cada sintoma depressivo aferido pela escala de depressão geriátrica de Yesavage, houve diminuição de $11 \%$ da probabilidade de o idoso do grupo ser fisicamente ativo.
\end{abstract}

Palavras-chave: Atividade física para idoso; Estresse psicológico; Depressão.

\begin{abstract}
This is a research carried out with a group of elderly people who actively participate in an Elderly Living Center located in the East Zone of São Paulo. The objective was to verify the association between the levels of physical activity and variables related both to the multidimensionality of health in the elderly and to the degree of perceived stress. It was also investigated which of the studied variables influenced the levels of physical activity found in this group. 57 elderly people participated, a majority of women, widowed or married, with low education levels, an average age 74.7 years, able to perform basic and instrumental activities of daily living independently, without risk of
\end{abstract}


falling or of dementia. These elders, in general, self-perceived their health as good or excellent, being physically active. A statistically significant association was found between the presence of depressive symptoms and high levels of perceived stress, noting that irregularly active elderly people had more depressive symptoms and worse selfperceived health. For each depressive symptom measured by the Yesavage geriatric depression scale, there was an $11 \%$ decrease in the probability of the elderly in the group being physically active.

Keywords: Physical activity for the elderly; Psychological stress; Depression.

\section{Resumen}

Se trata de una investigación realizada con un grupo de persona mayores que participan activamente en un Centro de Convivencia ubicado en la Zona Este de São Paulo. El objetivo fue verificar la asociación entre los niveles de actividad física y variables relacionadas tanto con la multidimensionalidad de la salud en la persona mayor como con el grado de estrés percibido. También se investigó cuál de las variables estudiadas influyó en los niveles de actividad física encontrados en este grupo. Participaron 57 personas mayores, en su mayoría mujeres, viudas o casadas, con baja escolaridad y edad promedio a los 74,7 años, independientes para el desempeño de actividades básicas e instrumentales de la vida diaria, sin riesgo de caídas o para demencias. Estas personas mayores, en general, auto perciben su salud como buena o excelente, siendo físicamente activas. Se encontró asociación estadísticamente significativa entre la presencia de síntomas depresivos y altos niveles de estrés percibido, destacando que los ancianos irregularmente activos tenían más síntomas depresivos y peor salud auto percibida. Para cada síntoma depresivo medido por la escala de depresión geriátrica de Yesavage, hubo una disminución del $11 \%$ en la probabilidad de que los ancianos del grupo fueran físicamente activos.

Palabras clave: Actividad física para personas mayores; Estrés psicológico; Depresión.

\section{Introdução}

O envelhecer fisiológico, processo denominado de senescência, acarreta inúmeras mudanças no organismo, tais como diminuição do peso corporal e da massa muscular, como exemplos. Estas modificações, esperadas para um corpo que envelhece, podem vir associadas ao aparecimento de enfermidades, o que prejudica a qualidade de vida das pessoas idosas (Almeida et al., 2020).

A população acima de 60 anos compõe, atualmente, a faixa etária com maior prevalência de Doenças Crônicas Não Transmissíveis (DCNT) -tais como Hipertensão Arterial Sistêmica e Diabetes Mellitus- sendo o sedentarismo considerado como um dos principais fatores de risco relacionado a estas enfermidades. Segundo a Organização Mundial de Saúde-OMS (World Health Organization (WHO), 2018), estima-se que 3,2 milhões de pessoas morrem a cada ano como consequência de DCNT relacionadas aos baixos níveis de atividade física. Pesquisa realizada pelo Instituto Brasileiro de Geografia e Estatística (Instituto Brasileiro de Geografia e Estatística (IBGE), 2017) concluiu que 71,4\% da população idosa brasileira não pratica nenhuma atividade física de maneira regular.

Com a senescência, alterações no aparelho locomotor podem levar à perda no equilíbrio, fragilidade óssea, presença de dores articulares e decréscimos da funcionalidade global, as quais podem ter seu efeito minimizado mediante a prática regular de exercícios físicos. Realizar exercícios relaciona-se ainda à melhoria das funções orgânicas e cognitivas, garantindo maior independência e prevenindo doenças, independentemente da idade de quem pratica (Almeida et al., 2020; Peixoto et al., 2018)

A OMS (WHO, 2018) considera como prática de atividade física, a execução regular de 150 minutos/semana, sendo que, em 2018, elaborou um plano de ação mundial visando reduzir em 15\% a inatividade física entre as pessoas idosas até 2030. A organização destaca que a prática de exercícios físicos por este segmento etário auxilia não apenas na prevenção de quedas, mas reduz o desencadeamento de síndrome de fragilidade e de imobilidade, minimiza o surgimento de incontinência urinaria e diminui sintomas relacionados a alterações de humor.

Os benefícios da atividade física à saúde são atualmente inegáveis, porém ainda há poucas pesquisas envolvendo a temática em pessoas idosas tendo como foco a relação desta com condições psíquicas, tais como o estresse. Assim, propôs-se este estudo, o qual buscou verificar a associação entre os níveis de prática de atividade física por um grupo de idosos 
frequentadores de Núcleo de Convivência para Idosos (NCI), localizado na cidade de São Paulo, e variáveis relacionadas tanto à multidimensionalidade da saúde na pessoa idosa quanto ao grau de estresse percebido. Investigou-se, ainda, quais das variáveis estudadas influenciaram os níveis de atividade física encontrados neste grupo.

\section{Metodologia}

Pesquisa quantitativa, exploratória, transversal, empreendido com um grupo de idosos moradores da cidade de São Paulo e que frequentavam ativamente um NCI localizado na Zona Leste da referida cidade, durante o ano de 2019.

Os NCI são serviços da área da Seguridade Social, caracterizados pelo seu papel de estímulo à convivência e fortalecimento de vínculos, voltados para pessoas acima de 60 anos que se encontram em situação de vulnerabilidade, risco pessoal e social, caracterizada pela presença de isolamento social e ausência de oportunidades de convívio familiar e comunitário. São locais onde se promove acessibilidade, trabalhos sociais e socioeducativos/culturais, que tornam o processo de envelhecimento mais ativo e saudável e geram motivação para novos projetos de vida, além de prevenir doenças, isolamento e asilamento. Na cidade de São Paulo, a maior parte dos idosos que atendem a estas condições residem nas periferias da cidade, com destaque para as Zonas Sul e Leste (Ministério do Desenvolvimento Social e Combate à Fome (MDCF), 2014).

Para este estudo foram incluídos todos os 57 idosos que frequentavam ativamente as oficinas propostas pelo NCI e não houve perdas. Antes da realização da pesquisa, estas pessoas idosas foram informadas do objetivo desta e, tendo concordado em participar, assinaram Termo de Consentimento Livre e Esclarecido. A pesquisa foi realizada em datas previamente combinadas com a gerência do NCI e com os idosos, em local disponibilizado pelo próprio serviço.

Para a coleta de dados, aplicou-se questionário sociodemográfico que incluiu as variáveis: idade, sexo, estado civil e escolaridade. A fim de verificar a multidimensionalidade da saúde destes idosos, foram aplicadas escalas, com pontos de corte e escores, preconizadas pelo Ministério da Saúde Brasileiro (Dias \& Rodrigues, 2015; Gomes \& Martins, 2015; Leis \& Manso, 2015). Desta forma, utilizaram-se: (i) escalas de Katz e Lawton para verificação do desempenho nas atividades básicas e instrumentais da vida diária, ABVD e AIVD, respectivamente; (ii) Teste Time Up And Go (TUG) para análise no risco de quedas; (iii) Miniexame do Estado Mental (MEEM) para avaliação de risco cognitivo para demências e (iv) Escala de Depressão Geriátrica EDG-30 de Yesavage para averiguação da presença de sintomas depressivos. Inqueriu-se ainda sobre a autopercepção de saúde, como também recomenda o Ministério da Saúde (Manso, Jesus, \& Gino, 2020).

O estresse percebido foi avaliado pela Escala de Estresse Percebido (Perceived Stress Scale -PPS), instrumento validado e adaptado para uso no Brasil. Trata-se de instrumento com 14 itens, com opções de resposta tipo Likert que variam de 0 a 4 , sendo $0=$ nunca e $4=$ sempre. Há questões com conotação positiva do estresse, as quais tem sua pontuação somada invertida; já as perguntas com conotação negativa, são somadas diretamente. Os escores totais podem variar de 0 a 56 , sendo que, quanto maior a pontuação, maior o nível de estresse percebido (Luft, Sanches, Mazo, \& Andrade, 2007). Ressaltam Pereira, Shitsuka, Parreira \& Shitsuka (2018) que, apesar de pesquisas que utilizem a escala Likert poderem ser consideradas qualitativas; "pode-se agrupar para cada questão, as respostas dos entrevistados e com a quantidade de respostas, por se possuir dados numéricos, pode-se realizar um tratamento estatístico" (p. 68).

A fim de mensurar o grau de atividade física praticado por estes idosos, optou-se pela utilização do Questionário Internacional de Atividade Física (IPAQ), validado transculturalmente para uso na população brasileira, sendo utilizada a versão adaptada para a população idosa. O questionário se baseia nas atividades de uma semana comum, investigadas por meio de 15 perguntas que versam sobre atividade física no trabalho, no meio de transporte, em casa, recreativa e tempo gasto 
sentado, associadas ao grau de frequência (em dias por semana) e duração (tempo). Ao término do questionário classifica-se as pessoas idosas conforme níveis de atividade física em muito ativos, ativos e irregularmente ativos (Mazo \& Benedetti, 2010).

Os dados, organizados em planilha de Excel, foram submetidos à análise estatística por meio do software SPSS versão 25 IBM®. Para a realização dos testes estatísticos, os resultados foram realocados em grupos conforme classificação das pessoas idosas segundo os níveis de atividade física mensurados pelo IPAQ. A comparação entre os grupos foi feita através do teste t-Student (variáveis quantitativas) e Qui-quadrado ou Exato de Fisher (variáveis qualitativas). A seguir, efetuou-se análise multivariada a fim de verificar as variáveis que interferem em ser ativo ou muito ativo. Foi considerado, para todos os testes, um nível de significância de 5\% ( $\mathrm{p}<0,05)$.

Este estudo teve sua realização aprovada pelo Comitê de Ética e Pesquisa sob número 2.626.054, CAAE: 87307118500005482 .

\section{Resultados}

O estudo contou com um total de 57 idosos: $50(87,70 \%)$ mulheres e sete $(12,30 \%)$ homens, com média de idade de 74,7 anos, variando entre 61 a 88 anos (mediana= 75 anos e $\mathrm{S}=6,8$ ). Observou-se predomínio de pessoas com escolaridade baixa, em sua maioria viúvos ou casados, como se observa na Tabela 1, onde são apresentadas as variáveis sociodemográficas do grupo pesquisado.

Tabela 1. Distribuição segundo variáveis sociodemográficas, grupo de idosos frequentadores do NCI, Zona Leste de São Paulo, 2019.

\begin{tabular}{lll}
\hline Variável & n & \% \\
\hline Estado civil & & \\
Viúvo(a) & 27 & 47,40 \\
Casado(a) & 19 & 33,30 \\
Solteiro(a) & 6 & 10,50 \\
Divorciado(a) & 5 & 8,80 \\
& & \\
Escolaridade & & \\
Fundamental incompleto & 37 & 64,90 \\
Fundamental completo & 11 & 19,30 \\
Analfabeto & 3 & 5,30 \\
Médio completo & 3 & 5,30 \\
Superior completo & 2 & 3,50 \\
Médio incompleto & 1 & 1,80 \\
\hline
\end{tabular}

Fonte: SPSS®, dados da pesquisa.

Quanto à autoavaliação de saúde, 29 (50,90\%) destas pessoas a consideraram como boa e 17 (29,80\%) como ótima. As variáveis relacionadas à capacidade funcional apresentam-se na Tabela 2. 
Tabela 2. Distribuição segundo variáveis relacionadas à capacidade funcional, grupo de idosos frequentadores do NCI, Zona Leste de São Paulo, 2019.

\begin{tabular}{|c|c|c|}
\hline Variável & $\mathbf{n}$ & $\%$ \\
\hline \multicolumn{3}{|l|}{$A B V D^{*}$} \\
\hline Independente & 36 & 63,20 \\
\hline Dependente & 21 & 36,80 \\
\hline \multicolumn{3}{|l|}{$A I V D^{* *}$} \\
\hline Independente & 56 & 98,20 \\
\hline Dependente & 1 & 1,80 \\
\hline \multicolumn{3}{|l|}{$T U G^{\dagger}$} \\
\hline Sem dificuldade & 37 & 64,90 \\
\hline Dificuldade leve a moderada (20-29 s) & 16 & 28,10 \\
\hline Dificuldade grave (> $30 \mathrm{~s})$ & 4 & 7,00 \\
\hline \multicolumn{3}{|l|}{$M E E M^{\dagger}$} \\
\hline Normal & 57 & 100 \\
\hline
\end{tabular}

*Atividades Básicas de Vida Diária; ** Atividades Instrumentais de Vida Diária; † Timed Up and Go; $\$$ Mini Exame Estado Mental Fonte: SPSS®, dados da pesquisa.

Quanto à EDG-30 itens, a pontuação média obtida foi de 8,4 $(\mathrm{S}=6,2)$, mediana de 7, valor mínimo 0 (ausência de sintomas depressivos) e máximo de 24. Para a PPS, os escores variaram de 0 a 51, com média de 19,74 (S=10,97) e mediana de 18. Apresentaram níveis baixos de estresse percebido (até 18) 29 idosos (50,90\%) e alto (> 18) 28 pessoas (49,10\%).

Classificadas as pessoas idosas do grupo segundo níveis de atividade física pelo instrumento IPAQ, obteve-se 43 $(75,40 \%)$ pessoas ativas e $13(22,80 \%)$ irregularmente ativas. Apenas um idoso foi considerado como muito ativo.

Quando efetuada a análise de associação, obteve-se que pessoas irregularmente ativas apresentavam mais sintomas depressivos e, portanto, maior pontuação na EDG ( $\mathrm{p}=0,013)$. Notou-se ainda que idosos ativos apresentaram melhor percepção de saúde $(\mathrm{p}=0,027)$. Não houve nenhuma associação significativa entre a escala PPS e os níveis de atividade física, porém observou-se que quanto maior a pontuação na escala PPS, maior a presença de sintomas depressivos $(\mathrm{p}=0,018)$.

Pela regressão logística, pode-se observar que a única variável avaliada que influencia ser ativo ou irregularmente ativo foi a presença de sintomas depressivos aferidos pela EDG, mostrando que para cada ponto a mais na EDG há diminuição de $11 \%$ na probabilidade de o idoso ser ativo.

\section{Discussão}

A distribuição sociodemográfica encontrada para este grupo se destaca pela presença de elevada média etária. O Brasil vem apresentando um rápido crescimento populacional, caracterizado por um maior incremento nas faixas etárias mais velhas, faixas onde as mulheres suplantam os homens. Ressalta-se ainda que a preponderância de mulheres no grupo é corroborada por outros estudos realizados no país que demonstram que mulheres, de elevada idade, predominantemente viúvas ou casadas e com baixa escolaridade são as que mais frequentam serviços públicos correlatos aos NCIs (Manso, D’Oliveira, \& Almeida, 2020; IBGE, 2015).

A saúde da pessoa idosa deve ser analisada de um ponto de vista multidimensional, em seus aspectos não apenas biológicos, mas psíquicos e sociais. A fim de apreender esta multidimensionalidade, vários instrumentos foram propostos, com destaque para a Avaliação Multidimensional da Pessoa Idosa ou AMI. Um dos componentes avaliados pela AMI refere-se à capacidade de execução de tarefas rotineiras sem auxílio (independência), outro diz respeito à manutenção da habilidade de 
tomar decisões e de gerir a própria vida (autonomia). Estes dois aspectos são itens extremamente importantes a serem preservados conforme se envelhece (OMS, 2015; Câmara, Gerez, Miranda, \& Velardi, 2008).

Estes dois aspectos- autonomia e independência- podem ser apreciados mediante a aplicação de escalas. Deste modo, avaliam-se as ABVD, funções relacionadas à sobrevivência tais como alimentar-se, ir ao banheiro, dentre outras. Já as AIVD traduzem problemas mais complexos, tais como gerir seu próprio dinheiro, fazer compras, sair sozinho de casa. A perda de independência e autonomia não só afetam gravemente a qualidade de vida da pessoa idosa, como também se relaciona à necessidade de cuidados a longo prazo (OMS, 2015; World Economic Forum, 2012).

Neste grupo pesquisado, já se observam pessoas idosas necessitando de cuidados relacionados ao desempenho das ABVD, estando as AIVD preservadas na grande maioria destes idosos. Não é o que a literatura destaca como sendo um padrão comum, posto que pesquisas demonstram que, no envelhecer patológico, primeiro se perdem habilidades relacionadas às AIVD (OMS, 2015). Este é um ponto que deve ser melhor estudado neste grupo.

Ainda como parte da AMI, o TUG é utilizado como preditor do risco de quedas, avaliando de modo cronometrado a potência, velocidade, agilidade e equilíbrio, mediante a análise da mobilidade funcional ao levantar-se de uma cadeira sem braços, caminhar por três metros, girar, voltar e se sentar. No presente estudo, mais da metade dos idosos participantes $(64,9 \%)$ não apresentaram dificuldades na realização do teste, como encontrado em outros estudos empreendidos com grupos semelhantes ao aqui descrito (Leis \& Manso, 2015; Campos, Vianna, \& Campos, 2013)

Por sua vez, o Mini Exame do Estado Mental (MEEM) é o teste de rastreio cognitivo mais utilizado em todo o mundo, cujo objetivo é avaliar o estado mental, mais especificamente sintomas de demência. No grupo estudado, nenhuma pessoa idosa apresentou risco, o que também se destacou em relação a outros grupos estudos semelhantes, onde, para um grupo de pessoas idosas com as características sociodemográficas semelhantes a este grupo, já se esperaria alguma alteração (Melo \& Barbosa, 2015).

Nenhuma destas variáveis até aqui expostas, associaram-se aos níveis de desempenho de atividade física neste grupo, os quais foram elevados quando comparados com a população idosa brasileira (IBGE, 2017), fato confirmado por outros estudos que demonstram que pessoas idosas que frequentam equipamentos tais como NCIs são mais ativos. Isto se deve às atividades que costumam ser desenvolvidas nestes espaços, as quais quase sempre incluem dança, yoga ou outro tipo de atividade física (Almeida, Souza, Rocha, Fernandes, Evangelista, Ribeiro, 2020; Santos, Silva, Reis, Rocha, \& Freitas, 2019).

Das variáveis estudadas e que se relacionaram com os graus de atividade física, a autopercepção de saúde é considerada um importante indicador sobre o bem-estar individual e qualidade de vida em idosos, revelando a concepção do indivíduo sobre a própria condição de saúde (Carneiro et al., 2020).

Vários estudos demonstram que, para a população geriátrica, a autopercepção de saúde permanece elevada mesmo em pessoas idosas com múltiplas enfermidades, pois se relaciona com a visão que estas pessoas possuem sobre seu desempenho: caso consigam realizar suas atividades rotineiras e viver de forma que avaliem como independente, a percepção tende a ser positiva (Manso, Jesus e Gino, 2020).

A autopercepção associa-se ainda, neste segmento populacional, à autoestima elevada e à prática regular de atividades físicas (Bortoluzzi et al., 2018; Ribeiro et al., 2018). Neste grupo, pessoas idosas ativas e muito ativas tiveram melhor autopercepção de saúde, como preconiza a literatura.

No que diz respeito ao estresse percebido, não foi encontrada associação estatística entre níveis de atividade física e escores na escala PPS. O estresse se caracteriza por ser uma relação singular entre a pessoa e o meio ambiente. Mediada por processos cognitivos, decorre de avaliação individual sobre uma dada a situação e os recursos de enfrentamento que a pessoa dispõe, podendo ser tida como ameaçadora ou não. Assim, considerar uma situação como estressora dependerá como o evento 
desencadeante é percebido e interpretado, podendo, inclusive, variar conforme a cultura, o momento histórico e o ambiente no qual o sujeito se insere (Lazarus, 1993).

Quando um evento é considerado estressor, o eixo hipotálamo-hipófise-adrenal é ativado e, caso este persista intensificado por um longo período, pode favorecer o desencadeamento/agravamento/descompensação de condições tais como as DCNT. Ainda, pode haver redução da imunidade e alteração do comportamento emocional, da função social, dos processos cognitivos e das crenças espirituais, gerando sofrimento e prejudicando a comunicação. Na pessoa idosa, que pode já apresentar redução da reserva funcional e cognitiva devido a senescência, pode levar à fragilização (Rocha et al., 2018).

Foram recuperados poucos artigos que interrelacionem estresse percebido e atividade física em idosos, porém, a prática regular parece diminuir o nível de estresse nas pessoas idosas (Santos et al., 2019), o que não foi notado para este grupo.

No que concerne aos escores de estresse observados para estes idosos, não foi encontrado nenhum estudo feito no Brasil especificamente com escores obtidos pela aplicação da escala PPS em pessoas idosas, o que dificulta comparar os resultados obtidos e demonstra uma lacuna de pesquisa. Para este grupo, encontrou-se ainda associação entre os graus de estresse percebido e sintomas depressivos, sendo que, quanto mais elevados os primeiros, maior a presença dos segundos.

Depressão é um transtorno de humor que pode interferir em todos os aspectos da vida de um indivíduo, considerado um problema médico grave entre as pessoas idosas e altamente prevalente. Dificuldades financeiras e de acesso a serviços essenciais, aposentadoria, morte de amigos e familiares, problemas com os filhos e netos, presença de violência e discriminação social, tornam os idosos mais susceptíveis tanto ao estresse quanto à depressão (Guimarães et al., 2019).

Dado que os fatores relacionados à depressão nas pessoas idosas também são considerados eventos estressores (Ramos et al., 2019), não causa estranheza o achado desta pesquisa que mostra que quanto maior a presença de sintomas depressivos, maior o escore na escala PPS

Neste grupo, pessoas idosas ativas e muito ativas tiveram menos sintomas depressivos. Estudos apontam que o exercício físico reduz significativamente o estresse, além de aumentar as endorfinas no sistema nervoso, sendo assim capaz de reduzir e/ou prevenir a depressão. Além do impacto direto em nível central, a prática de atividade física também auxilia na prevenção e manejo de doenças crônicas, fator que, entre as pessoas idosas, é importante para definir suas limitações e independência e, consequentemente, seu estado mental (Guimarães et al., 2019; Hernandes \& Voser, 2019).

Por fim, cabe destacar a influência dos sintomas depressivos sobre a prática de atividade física. Para este grupo de pessoas, cada ponto a mais na escala EDG, traduzindo a presença de sintomas depressivos, diminuiu em $11 \%$ a possibilidade de o idoso ser fisicamente ativo, demonstrando a presença de um possível círculo vicioso, onde a depressão restringe a prática física e esta deixa de ativar mediadores neurológicos importantes que poderiam diminuir a presença de depressão.

Estudos psicológicos demonstram a presença, em pessoas idosas, do que se denomina espiral depressiva. Esta caracteriza-se pela intrusão de pensamento negativos, os quais seriam substituídos, pela prática de atividade física, por pensamentos positivos, o que melhoraria o quadro depressivo. Realizar alguma prática física relaciona-se ainda com a melhora da autoestima, o convívio social e pode proporcionar distração para eventos estressores cotidianos, o que se relaciona com melhora dos sintomas depressivos (Hernandes \& Voser, 2019; Gabriel \& Pozzobon, 2013).

\section{Considerações Finais}

O grupo de idosos pesquisado apresentou níveis de atividade física elevados em relação à população idosa brasileira, não sendo encontrada associação com a maioria das variáveis relacionadas à multidimensionalidade da avaliação de saúde do grupo, exceto para sintomas depressivos e autopercepção de saúde. Não se encontrou relação entre os níveis de estresse 
percebido e a prática física, porém pessoas classificadas com escores de atividade física pelo IPAQ como ativos e muito ativos apresentaram melhor autopercepção de saúde e menor pontuação na escala EDG.

Acredita-se que o tamanho da amostra foi um limitador importante para esta pesquisa, aventando-se a hipótese de que, caso o estudo seja repetido com um número maior de pessoas idosas, se possa demonstrar a relação entre níveis de atividade física e a presença de estresse avaliado pela escala PPS. Nesta pesquisa, apesar da correlação não ser encontrada, ficou clara a associação com estresse-depressão e o quanto a presença de sintomas depressivos afeta a prática da atividade física.

Este achado aponta para a necessidade de maior atenção a este grupo de pessoas idosas com sintomas depressivos, pois sabe-se que a prática regular de atividades físicas diminui e/ou retarda os níveis de estresse e depressão, acarretando melhora na qualidade de vida e na autopercepção de saúde.

A pesquisa trouxe ainda um ponto interessante para aprofundamento posterior: a dependência deste grupo de idosos em relação às ABVD e independência em relação às AIVD, como comentado, achado que difere do citado pela literatura.

Outro ponto a ser ressaltado diz respeito ao próprio grupo, o qual, apesar de ter características sociodemográficas semelhantes a outros grupos de idosos pesquisados e que frequentam equipamentos públicos análogos, estão sujeitos a questões sócio-históricas singulares, dificultando comparações. Acredita-se, entretanto, que a pesquisa atingiu seus objetivos, demonstrando a importância da relação entre aspectos psíquicos, tais como estresse e depressão, com a prática de atividade física por pessoas idosas.

\section{Referências}

Almeida, B. L., Souza, M. E. B. F., Rocha, F. C., Fernandes, T. F., Evangelista, C. B., Ribeiro, K. S. M. A. (2020) Qualidade de vida de idosos que praticam atividade física. Revista Funcional Care, 12, 466-470.

Bortoluzzi, E. C. et al. (2018). Autopercepção de saúde de idosas praticantes de atividades físicas e fatores associados. Estudos interdisciplinares envelhecimento, 23 (2), 119-131

Câmara, F. M., Gerez, A. G., Miranda, M. L. J., \& Velardi, M. (2008). Capacidade funcional do idoso: formas de avaliação e tendências. Acta fisiátrica, $15(4), 249-256$

Campos, M. S., Vianna, L. G., \& Campos, A. R. (2013). Os testes de equilíbrio, alcance funcional e "Timed Up and Go" e o risco de quedas em idosos. São Paulo: PUC-SP: Revista Kairós-Gerontologia, 16(4), 125-138.

Carneiro, J.A. et al. (2020) Autopercepção negativa da saúde: prevalência e fatores associados entre idosos assistidos em centro de referência. Ciência \& Saúde Coletiva, 25 (3): 909-918

Dias, A. L., \& Rodrigues, T. C. (2015). Avaliação da Capacidade Funcional. In: Manso, M. E. G. \& Biffi, E. C. A. (Orgs.). Geriatria, Manual da Liga de Estudos do Processo de Envelhecimento. São Paulo, SP: Martinari.

Gabriel, K. \& Pozzobon, A. (2013). Efeito da atividade física na depressão e na qualidade de vida de idosos. Cinergis, 14(2), 134-137. $10.17058 /$ cinergis.v14i2.3643

Gomes, L. M. C., \& Martins, L. N. S. L. (2015). Avaliação Cognitiva e do Humor. In: Manso, M. E. G., \& Biffi, E. C. A. (Orgs.). Geriatria, Manual da Liga de Estudos do Processo de Envelhecimento. Martinari.

Guimarães, L. A. et al. (2019). Sintomas depressivos e fatores associados em idosos residentes em instituição de longa permanência. Ciência \& Saúde Coletiva, 24 (9): 3275-3282

Hernandez, J. A. E. \& Voser, R. C. (2019). Exercício físico regular e depressão em idosos. Estudos e pesquisa em Psicologia. https://www.epublicacoes.uerj.br/index.php/revispsi/article/view/46912/31302

Instituto Brasileiro de Geografia e Estatística. IBGE. (2015). Uma Análise das Condições de Vida da População Brasileira 2010. http://www.ibge.gov.br/home/estatistica/populacao/condicaodevida/indicadoresminimos/sinteseindicsociais2010/SIS_2010.pdf.

Instituto Brasileiro de Geografia e Estatística. IBGE. (2017). Práticas de esporte e atividade física. Pesquisa Nacional por Amostra de Domicílios (PNAD). Rio de Janeiro: IBGE.

Lazarus, R. S. (1993) Coping theory and research: Past, present, and future. Psychosomatic Medicine, 55: $234-247$.

Leis, D. F., \& Manso, M. E. G. (2015). Avaliação da Marcha, Equilíbrio e Atividade Física no idoso. In: Manso, M. E. G., \& Biffi, E. C. A. (Orgs.). Geriatria, Manual da Liga de Estudos do Processo de Envelhecimento. Martinari. 
Research, Society and Development, v. 10, n. 1, e12710111612, 2021 (CC BY 4.0) | ISSN 2525-3409 | DOI: http://dx.doi.org/10.33448/rsd-v10i1.11612

Luft, C. D. B., Sanches, S. O., Mazo, G. Z., \& Andrade, A. (2007). Versão brasileira da Escala de Estresse Percebido: tradução e validação para idosos. Revista de Saúde Pública, 41 (4), 606-615.

Manso, M. E. G., D’Oliveira, R. R., \& Almeida, V. P. (2020). A triangulação de métodos na abordagem da violência contra o idoso: resultados em um núcleo de convivência. Research, Society and Development, 9 (10): e1409108522, 2020. http://dx.doi.org/10.33448/rsd-v9i10.8522

Manso, M. E. G., Jesus, L. S., \& Gino, D. R. (2020). Self-perceived health in a group of older adults covered by a health insurance plan. Geriatrics, Gerontology and Aging. 14(2):91-7

Maso, G. Z. \& Benedetti, T. R. B. (2010). Adaptação do questionário internacional de atividade física para idosos. Revista Brasileira Cineantropometria e Desempenho Humano. 12(6):480-4.

Melo, D. M. \& Barbosa, A. (2015). O uso do Mini Exame do Estado Mental em pesquisas com idosos no Brasil: uma revisão sistemática. Ciência \& Saúde Coletiva, 20 (12): 3865-3876.

Ministério do Desenvolvimento Social e Combate à Fome (2014). Tipificação Nacional de Serviços Socioassistenciais. MDS: Brasília.

OMS. (2015). Organização Mundial de Saúde. Relatório Mundial de Envelhecimento e Saúde. Genebra, Suíça: OMS http://apps.who.int/iris/bitstream/10665/186463/1/9789240694811_eng.pdf.

Pereira, A. S., Shitsuka, D. M., Parreira, F. J. \& Shitsuka, R. (2018) Licenciatura em computação. Metodologia da pesquisa científica. https://repositorio.ufsm.br/bitstream/handle/1/15824/Lic_Computacao_Metodologia-Pesquisa-Cientifica.pdf?sequence=1

Peixoto, S. V. et al. (2018). Physical activity practice among older adults: results of the ELSI-Brazil. Revista de Saúde Pública, 52 Suppl 2:5s

Ramos, F. P. et al. (2019). Fatores associados à depressão em idoso. Revista Eletrônica Acervo Saúde. (19): e239. https://doi.org/10.25248/reas.e239.2019

Ribeiro, E. G., Matozinhos, F. P., Guimarães, G. L., Couto, A. M., Azevedo, R. S., Mendoza, I. Y. Q. (2018). Self-perceived health and clinical functional vulnerability of the elderly in Belo Horizonte/Minas Gerais. Revista Brasileira de Enfermagem, 71(suppl 2):860-7.

Rocha, T. P. O. et al. (2018). Anatomofisiologia do estresse e o processo de adoecimento. Revista Científica FMC, 13 (2): $31-37$.

Santos, F. A. et al. (2018). Efeitos do exercício físico sobre o estresse percebido de idosos. Revista Interdisciplinar de Promoção da Saúde, 1 (2): 127-136

Santos, L. P., Silva, J. M. C. S., Reis, V. M. C. P., Rocha, J. S. B., \& Freitas, R. F. (2019). Nível de atividade física de idosos participantes de grupo de convivência e fatores associados. RBPFEX - Revista Brasileira de Prescrição e Fisiologia do Exercício, 13(83), 459-466.

World Economic Forum. (2012). Global Population Ageing: Peril or Promise? http://www.hsph.harvard.edu/pgda/working.htm.

World Health Organization. WHO. (2018). Physical activity. https://www.who.int/en/news-room/fact-sheets/detail/physical-activity 